\title{
Antibiotic sensitivity patterns in childhood urinary tract infections
}

\author{
A S Abeyagunawardena ${ }^{1}$, R S Thalgahagoda ${ }^{2}$, C A Pathinayake ${ }^{2}$, C K Abeysekera ${ }^{3}$
}

Sri Lanka Journal of Child Health, 2006; 35: 55-60

(Key words: urinary tract infections, antibiotic sensitivity pattern, renal scarring)

\begin{abstract}
Objectives To assess the current antibiotic sensitivity pattern of urinary pathogens and compare it with the pattern 5 years previously.
\end{abstract}

Method A retrospective analysis was performed on 2650 urine samples in 1997 and 2062 samples in 2002 received by the medical laboratory at the Teaching Hospital, Peradeniya from the paediatric ward and paediatric clinics.

Results In 1997 there were 155 urine culture reports with significant colony counts of $>10^{5}$ for which ABSTs were performed. 111 were from males and 44 from females. In 2002 there were 278 positive cultures of which 179 were from males and 99 from females. Predominant organism was the coliform, accounting for $90 \%$ of isolates in both 1997 and 2002. In 1997 nalidixic acid and nitrofurantoin had high sensitivities of $73.8 \%$ and $73.1 \%$ respectively, while ciprofloxacin and mecillinam had low sensitivities of $51.9 \%$ and $35.5 \%$. In 2002 coamoxiclav had the highest antibiotic sensitivity of $86.7 \%$, while nitrofurantoin, nalidixic acid and norfloxacin had high sensitivities of $80 \%, 76.6 \%$ and $75.7 \%$ respectively. Ciprofloxacin and mecillinam had low sensitivity levels both in 1997 and 2002. A change was seen in cotrimoxazole and cephalexin, which had low sensitivities of $40.2 \%$ and $54.8 \%$ in 1997 and relatively higher sensitivities of $63.8 \%$ and $69.2 \%$ in 2002 respectively.

\section{Introduction}

Urinary tract infections (UTIs) are commonly encountered in paediatric clinical practice accounting for $4.1-7.5 \%$ of febrile illnesses in childhood ${ }^{1}$. Apart from causing serious acute illness, UTIs can result in

\footnotetext{
${ }^{1}$ Senior Lecturer and Consultant Paediatrician, ${ }^{2}$ Assistant Temporary Lecturer, ${ }^{3}$ Senior Lecturer, Head and Consultant Paediatrician, Department of Paediatrics, Faculty of medicine, University of Peradeniya
}

(Received on 4 September 2005) renal scarring which may lead to hypertension and chronic renal failure in later life.

In children presenting with UTI, 30-40\% have underlying vesico-ureteric reflux (VUR) ${ }^{2}$, while other congenital anomalies like posterior urethral valves, pelvi-ureteric junction obstruction, ureteroceles and duplex systems are encountered less frequently.

The commonest organism isolated from urine cultures in children with UTI is Escherichia coli, accounting for $80-90 \%$ of childhood UTIs ${ }^{3,4}$. Other colonic bacteria like Klebsiella, Proteus and Pseudomonas are isolated less frequently. The commonest organisms isolated tend to vary from place to place, and thus the organisms present in a particular area will have a specific antibiotic sensitivity pattern.

The antibiotic sensitivity pattern of a particular organism is known to change with time ${ }^{5,6}$. Probable causes for this are continued usage of the same antibiotic, irrational usage of antibiotics and usage of inadequate dosages for an inadequate duration for treatment. A positive correlation between antibiotic usage and development of resistance has been observed in some studies ${ }^{7}$.

Treatment of the acute episode is of utmost importance as continuing infection can lead to renal scarring with potential progression to end stage renal failure later in life. Renal scarring has been shown to occur even after a single episode of pyelonephritis ${ }^{8,9}$ though the likelihood of scarring increases with recurrent episodes ${ }^{10,11}$. Therefore the prompt institution of the appropriate antibiotic therapy is essential.

In light of this, in most settings, antibiotic treatment of the acute episode of a febrile UTI is instituted prior to laboratory confirmation through a urine culture. As a result, the choice of the initial antibiotic is empirical. The antibiotic sensitivity pattern of the causative organisms should therefore be known in order to ensure that the most appropriate antibiotic is prescribed. A delay in administration of the 
appropriate antibiotic will increase the potential for renal scarring. It is imperative to emphasize that reflux nephropathy accounts for around $5-10 \%$ of end stage renal disease ${ }^{12,13}$ and therefore prevention of this is of utmost importance.

This study was undertaken to ascertain current antibiotic sensitivity pattern of urinary pathogens in a tertiary care setting and observe the changes in sensitivity pattern over a five year period.

\section{Method}

A retrospective analysis was made of all urine culture reports received by the medical laboratory at Teaching Hospital, Peradeniya from children admitted to the paediatric ward and from children attending paediatric clinics. All subjects included in study were $<12$ years of age. Two time segments of 1997 and 2002 were studied in order to make a comparative analysis of the two periods.

All cultures had been performed in the medical laboratory at Teaching Hospital, Peradeniya and the laboratory in the Department of Microbiology,
University of Peradeniya. Cystene Lactose Electrolyte Deficient Agar was used as the culture medium and the samples were incubated overnight at $35-37^{\circ} \mathrm{C}$. An antibiotic sensitivity test (ABST) had been performed on colony counts $>10^{5}$ which were deemed to be significant. Sensitivity of colonising organisms to nalidixic acid, nitrofurantoin, cotrimoxazole, norfloxacin, cephalexin ciprofloxacin, mecillinam, ampicillin, gentamicin, co-amoxyclav and cefradine, was assessed.

\section{Results}

A total of 2650 culture reports were analysed during 1997 of which $168(8.3 \%)$ had significant colony counts of or $>10^{5}$. During the year 2002, a total of 2062 culture reports were analysed of which 275 $(10.4 \%)$ had colony counts of or more than $10^{5}$. Of the 168 reports with siginficant growth in 1997 , $111(66 \%)$ were from males and $57(34 \%)$ were from females. The corresponding figures for 2002 were males $179(65 \%)$ and female $99(35 \%)$. The observed antibiotic sensitivity patterns are shown in Table1 and Figure 1.

Table 1

Antibiotic sensitivity pattern

\begin{tabular}{|l|c|c|}
\hline \multicolumn{1}{|c|}{ Antibiotic } & 1997 Sensitivity\% & 2002 Sensitivity \% \\
& & \\
\hline Nalidixic acid & $73.8(93 / 126)$ & $76.6(193 / 252)$ \\
\hline Nitrofurantoin & $73.2(109 / 149)$ & $80.0(212 / 264)$ \\
\hline Cotrimoxazole & $40.2(49 / 122)$ & $63.8(30 / 47)$ \\
\hline Norfloxacin & $67.0(79 / 118)$ & $75.7(28 / 37)$ \\
\hline Cephalexin & $54.8(17 / 31)$ & $69.2(148 / 214)$ \\
\hline Ciprofloxacin & $51.9(14 / 27)$ & $47.4(72 / 152)$ \\
\hline Mecillinam & $35.5(11 / 31)$ & $33.8(75 / 222)$ \\
\hline Ampicillin & $58.3(7 / 12)$ & $58.1(18 / 31)$ \\
\hline Gentamicin & $50.0(5 / 10)$ & $60.7(17 / 28)$ \\
\hline Co-Amoxyclav & - & $86.7(13 / 15)$ \\
\hline
\end{tabular}




\section{Antibiotic sensitivity pattern}

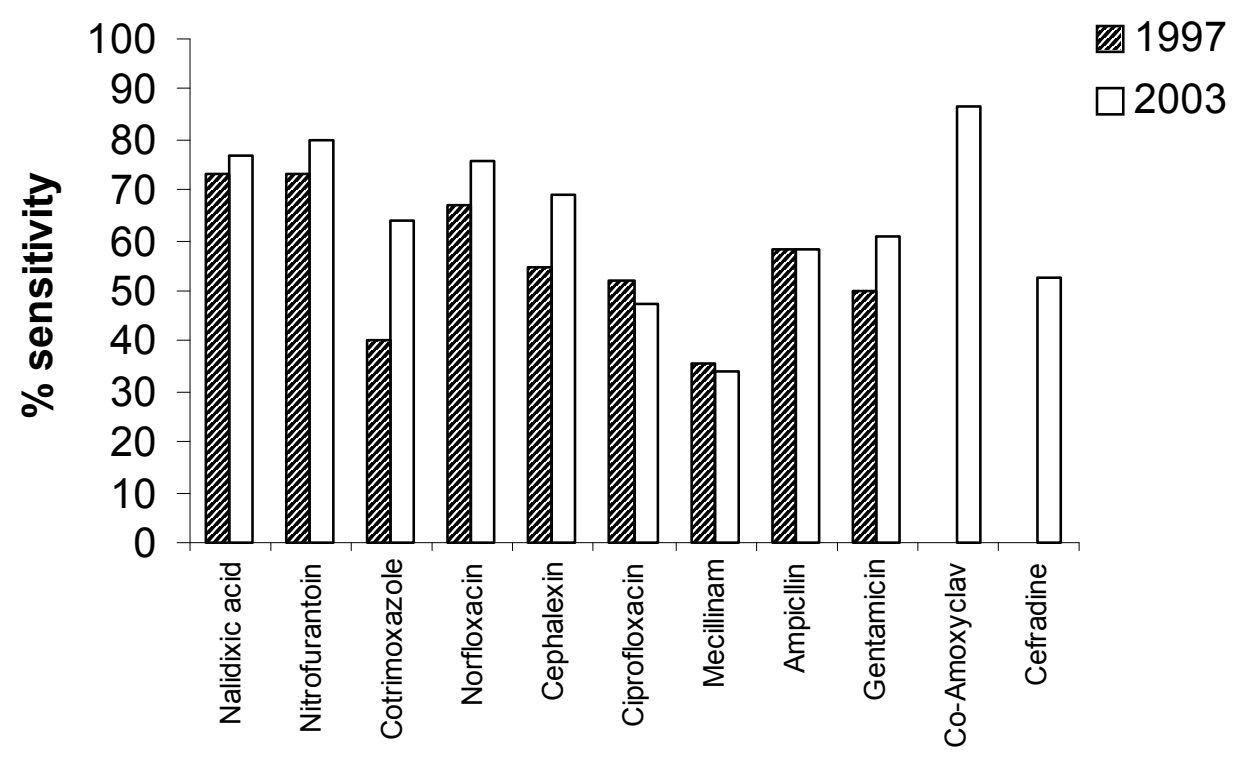

Figure 1

In 1997 nalidixic acid and nitrofurantoin had high sensitivities of $73.8 \%$ and $73.2 \%$ respectively while ciprofloxacin and mecillinam had low sensitivities of $51.9 \%$ and $35.5 \%$.

In 2002 co-amoxiclav had the highest antibiotic sensitivity of $86.7 \%$, while nitrofurantoin, nalidixic acid and norfloxacin also had high sensitivities of $80 \%, 76.6 \%$ and $75.7 \%$ respectively. Ciprofloxacin and mecillinam had low sensitivity levels of $47.4 \%$ and $33.8 \%$ in 2002 too.

A change was evident in the sensitivity levels of cotrimoxazole and cephalexin, which had low sensitivities of $40.2 \%$ and $54.8 \%$ respectively in 1997 and relatively higher sensitivities of $63.8 \%$ and $69.2 \%$ respectively in 2002 . The difference between the two years was significant when the statistical test to compare the two proportions was applied ( $\mathrm{P}$ value $0.03<0.05$ ). Antibiotic sensitivity tests had not been performed for co-amoxyclav and cefradine in 1997 and therefore a comparison was not possible.

\section{Discussion}

Ever since their discovery more than 50 years ago, antibiotics have been increasingly used to treat patients with bacterial infections. Today, they are vital for the treatment of bacterial infections, but increasing numbers of antibiotic resistant bacteria have progressively eroded their efficacy. Choosing an antibiotic from the wide range available, can present a dilemma for prescribers. The difficulty is heightened by the variations in sensitivity patterns during different periods of time. The degree of exposure of a population to specific antibiotics could play a role in this variation ${ }^{12,13}$.

Studies have shown that the acute inflammatory response caused by bacterial infection in pyelonephritis is responsible for renal parenchymal damage, which in turn leads to renal scarring ${ }^{14}$. In pyelonephritis, the bacterial inoculation of the renal parenchyma leads to complement activation by the bacterial lipopolysaccharides. This causes chemotactic migration of granulocytes to the area of infection leading to phagocytosis of the bacteria. With the killing of the bacteria by the granulocytes toxic enzymes are released within the granulocyte and also into the renal parenchyma causing renal cell damage ${ }^{15}$. The respiratory burst occurring at this time leads to the release of oxygen free radicals, which are toxic not only to the bacteria but also to the granulocytes and the surrounding renal tubular cells $^{16}$. The death of the tubular cells leads to the release of toxic inflammatory agents causing further cell damage. Bacterial infection of the renal parenchyma causes tissue ischaemia due to intravascular granulocyte aggregation and oedema. Re-perfusion of this ischaemic tissue leads to further 
production free radicals through cell reaction that leads to cellular damage ${ }^{17}$. Therefore it appears that toxic enzymes released during the acute inflammatory reaction as well as free radicals produced during re-perfusion of ischaemic tissue collaborate to cause the tissue damage that finally leads to renal scarring.

It is therefore clear that bacterial infection of the renal parenchyma is an essential prerequisite for the development of acquired renal scarring. In this view, prompt administration of the most appropriate antibiotic to treat the acute infection is vital in reducing the risk of renal scarring.

For the acute episode antibiotics are given either orally or intravenously, the choice being dependent on the age of the patient and the presence of features suggestive of renal parenchymal involvement such as loin pain, renal angle tenderness and fever of $>38.5^{\circ} \mathrm{c}$. Parenteral antibiotics are indicated for toxic and ill children with UTI at risk of developing renal scarring. The commonest antibiotics used for treatment of the acute episode include cephalosporins, co-amoxyclav, gentamicin and mecillinam, which have good tissue penetration capabilities.

The use of prophylactic antibiotics is indicated in patients at risk of developing recurrent infections due to underlying urinary tract abnormality in order to reduce the risk of renal scarring ${ }^{19,20}$. Prophylactic antibiotics are also prescribed until the completion of imaging of the urinary tract. The use of prophylactic antibiotics could reduce risk of the development of new renal scars by keeping the patient free of infection ${ }^{21,22}$. The prophylactic agent therefore needs to have a high level of sensitivity to prevent breakthrough infections. Nitrofurantoin, nalidixic acid and cotrimoxazole are the antibiotics commonly prescribed for prophylaxis. These agents, as well as ciprofloxacin and norfloxacin, are used for the treatment of lower urinary tract infections.

The results of this study indicate that nalidixic acid and nitrofurantoin have retained their high levels of sensitivity over a period of 5 years and therefore remain suitable for the treatment of lower urinary tract infections and for prophylaxis. Norfloxacin, with a high degree of sensitivity, is also suitable for the treatment of lower urinary tract infections. However ciprofloxacin and mecillinam had a low level of sensitivity indicating that resistance had built up against these two drugs and prescription of these two drugs empirically is therefore not recommended for the treatment of acute childhood UTI.

Co-amoxyclav, which had the highest level of sensitivity, along with the cephalosporins, remain the most suitable tissue penetrating antibiotics for the treatment of acute pyelonephritis. It was unfortunate that sensitivity levels for cefuroxime and cefotaxime had not been assessed due to the unavailability of discs, as these are commonly used antibiotics for the acute episode. Gentamicin, which had a low level of sensitivity in 1997, had attained a somewhat acceptable sensitivity level in 2002. The use of gentamicin for the treatment of the acute episode has diminished over the last few years due to its potential nephrotoxicity and also due to the availability of safer alternatives such as cephalosporins. It is this decrease in usage that is the most likely reason for its increased sensitivity in 2002 .

Cotrimoxazole and cephalexin, which had a low sensitivity in 1997, had attained acceptable sensitivities in 2002. The difference in sensitivity for cotrimoxazole did not reach acceptable statistical significance because the sample analyzed in 2002 was rather small. But this difference appears to be clinically significant. Cotrimoxazole, which was used frequently in childhood UTI in the past, has also diminished in recent times probably due to the fear of adverse effects of the sulphonamide component of cotrimoxasole. This decrease in usage may have contributed to cotrimoxazole achieving an acceptable sensitivity. It can now be considered for the treatment of lower UTI and for prophylaxis.

The change in the sensitivity of cotrimoxazole and gentamycin over the 5-year period is noteworthy. This emphasizes the importance of the periodic revision of antibiotic sensitivity patterns to facilitate treatment of the acute episode of childhood UTI with the most appropriate antibiotic in order to reduce the risk of renal scarring.

\section{Conclusion}

This study clearly indicates the need for continuous surveillance of the prevalence and antibiotic sensitivity pattern of microorganisms in the local environment, which should be the basis for effective empirical antibiotic therapy

\section{References}

1. Rushton HG. Urinary tract infections in children. Pediatric Clinics of North America 1997; 44:1133-69. 
2. International Reflux Study Committee. Medical versus surgical treatment of primary vesicoureteral reflux. Pediatrics 1981; 67: 392-400.

3. Jodal U, Winberg J. Management of children with unobstructed urinary tract infection. Pediatr Nephrol 1987; 1: 647.

4. Svanborg-Eden C, de Man P. Bacterial virulence in the urinary tract. Infect Dis Clin North Am 1987; 1: 731-50.

5. Wilina Lim, Khin Khin Tint, Ng TK, Tsang DNC and Que TL Current status of bacterial resistance to antibiotics in Hong Kong. $J$ Hong Kong Med Assoc 1991; 43: 152-8.

6. Adeyemo AA, Gbadegesin RA, Onyemenem TN, Ekweozor CC. Urinary tract pathogens and antimicrobial sensitivity patterns in children in Ibadan, Nigeria.Ann Trop Paediatr. 1994; 14(4): 271-4

7. Howard AJ et al. Factors associated with antibiotic resistance in coliform organisms from community urinary tract infection in Wales. J Antimicrob Chemother 2001; 47(3): 305-13.

8. Ransley PG, Risdon RA. Reflux nephropathy: effects of antimicrobial therapy on the evolution of the early pyelonephritic scar. Kidney Int 1981; 20: 733.

9. Smellie JM, Ransley PG, Normand ICS, et al. Development of new renal scars: a collaborative study. BMJ 1985; 290: 1957.

10. Smellie JM, Normand ICS, Katz G. Children with urinary tract infection: a comparison of those with and those without vesicoureteral reflux. Kidney Int 1981; 20: 717.

11. Winberg J, Bergstom $\mathrm{T}$, Jacobsson B. Morbidity, age, and sex distribution, recurrences and renal scarring in symptomatic urinary tract infection in childhood. Kidney Int 1975; 8: 100-1.

12. Arant BS Jr. Vesicoureteric reflux and renal injury. Am J Kidney Dis 1991; 17: 491.
13. Salvatierra O, Tanagho EA. Reflux as a cause of end stage kidney disease: report of 32 cases. J Urol 1977; 117: 441.

14. Pape L, Gunzer F, Ziesing S, Pape A, Offner $\mathrm{G}$ et.al. Bacterial pathogens, resistance patterns and treatment options in community acquired paediatric urinary tract infection. Klin Pediatr 2004; 216(2):83-6

15. Roberts JA. Mechanisms of renal damage in chronic pyelonephritis. Curr Top Pathol 1995; 88: 265-87.

16. Roberts JA, Roth JK, Dominque G, et al. Immunology of pyelonephritis in the primate model.V. Effect of superoxide dismutase. J Urol 1982; 128: 1394.

17. Kaack MV, Dowling KJ, Patterson GM, et al. Immunalogy of pyelonephritis. VIII. E. coli causes granulocyte aggregation and renal ischemia. J Urol 1986; 136:117.

18. American Academy of Paediatrics Committee on quality Improvement, Subcommittee on urinary tract infections. Practice parameter: The diagnosis, treatment and evaluation of the initial urinary tract infection in febrile infants and young children. Paediatrics 1999; 103:843-52.

19. Rushton HG. The evaluation of acute pyelonephritis and renal scarring with ${ }^{99 m}$ Tc-DMSA acid renal scintigraphy: evolving concepts and future directions. Pediatr Nephrol 1997; 11:108-20.

20. Jakobsson B, Berg U, Svensson L. Renal scarring after acute pyelonephritis. Arch Dis Child 1994; 70:111-5.

21. Smellie JM, Gruneberg RN, Bantock HM, et al. Prophylactic co-trimoxazole and trimethoprim in the management of urinary tract infection in children. Pediatr Nephrol 1988; 2:12-7.

22. Edwards D, Normand ICS, Prescod N, et al. Disappearance of vesicoureteric reflux during long-term antibiotic prophylaxis of urinary tract infection. BMJ 1977; 2: 285. 